\title{
Power Switch Device
}

National Cancer Institute

\section{Source}

National Cancer Institute. Power Switch Device. NCI Thesaurus. Code C50200.

A switch designed to regulate the power to a device. 